\title{
Archéopages
}

Archéopages

Archéologie et société

Hors-série 3 | 2012

Nouveaux champs de la recherche archéologique

\section{L'architecture des bâtiments du Néolithique récent final}

Ivan Praud

\section{OpenEdition}

1 Journals

Édition électronique

URL : https://journals.openedition.org/archeopages/694

DOI : 10.4000/archeopages.694

ISSN : 2269-9872

Éditeur

INRAP - Institut national de recherches archéologiques préventives

Édition imprimée

Date de publication : 1 janvier 2012

Pagination : 110-113

ISSN : $1622-8545$

\section{Référence électronique}

Ivan Praud, «L'architecture des bâtiments du Néolithique récent final », Archéopages [En ligne], Horssérie 3 | 2012, mis en ligne le 01 janvier 2012, consulté le 27 février 2023. URL : http:// journals.openedition.org/archeopages/694; DOI : https://doi.org/10.4000/archeopages.694

Tous droits réservés 


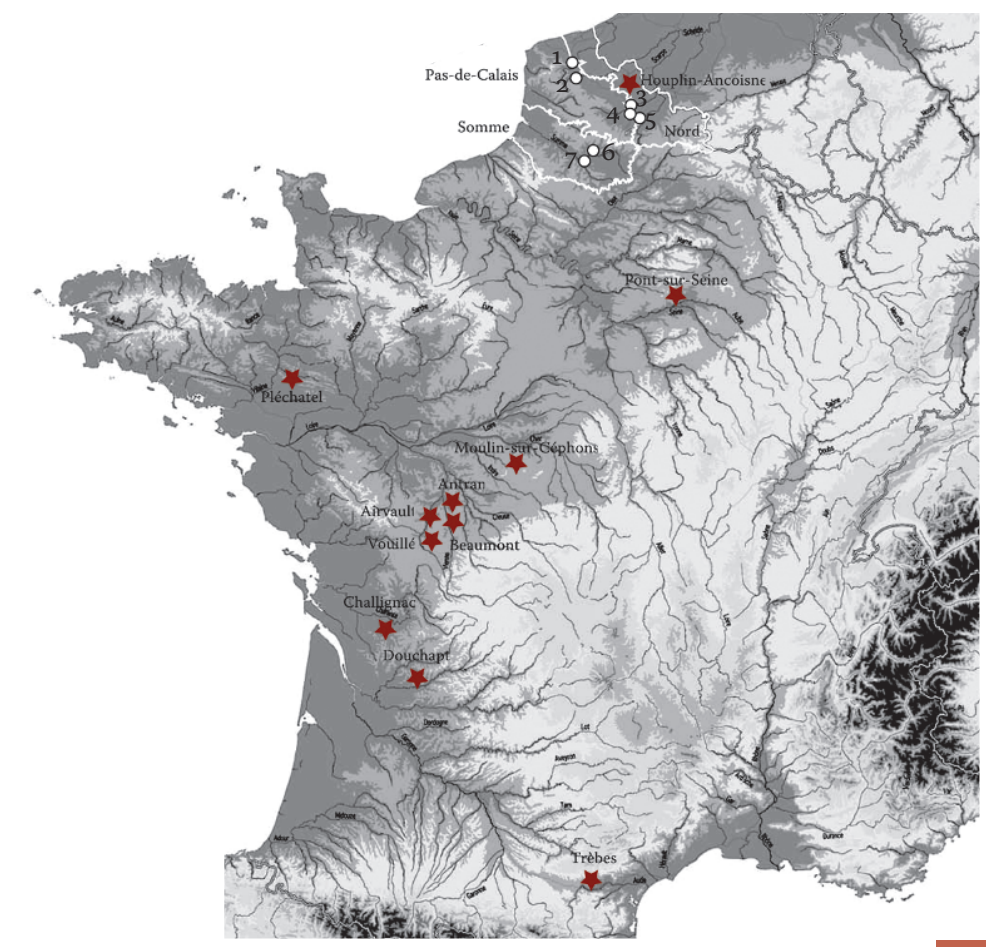

Les grands bâtiments

du Néolithique Récent Final

Les sites du Nord, du Pas-de-Calais,

et de la Somme :

1- Arcques

2- Aire-Sur-La-Lys

3- Lauwin-Planque « les Hussards »

4- Lambres-Lez-Douai

5- Arleux

6- Méaulte

7- Glisy

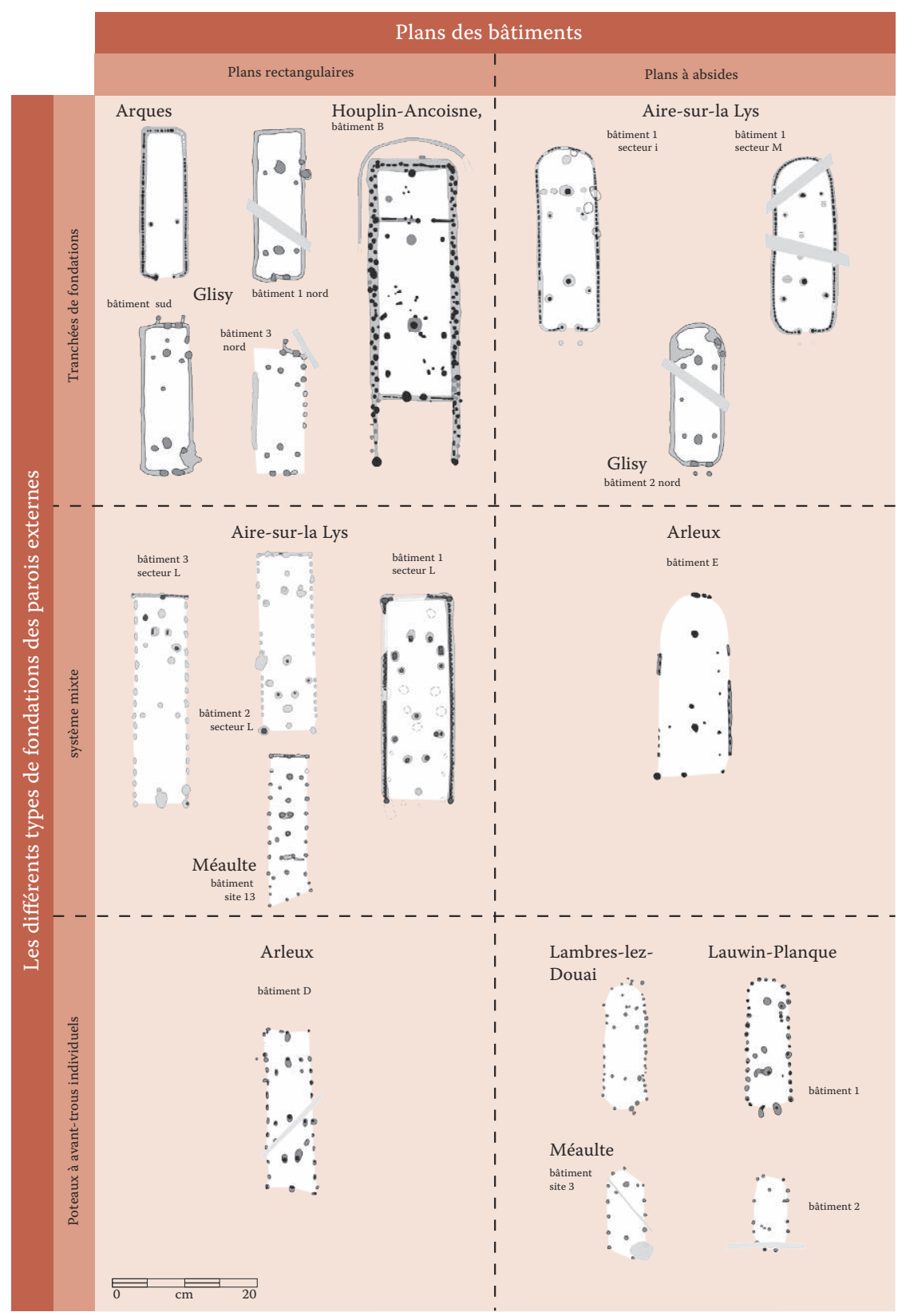

[Fig.1] Répartition des grands bâtiments du Néolithique récent final et localisation de l'aire d'étude des sites du groupe du Deûle-Escaut.

[Fig.2] Les plans des bâtiments du groupe Deûle-Escaut. 

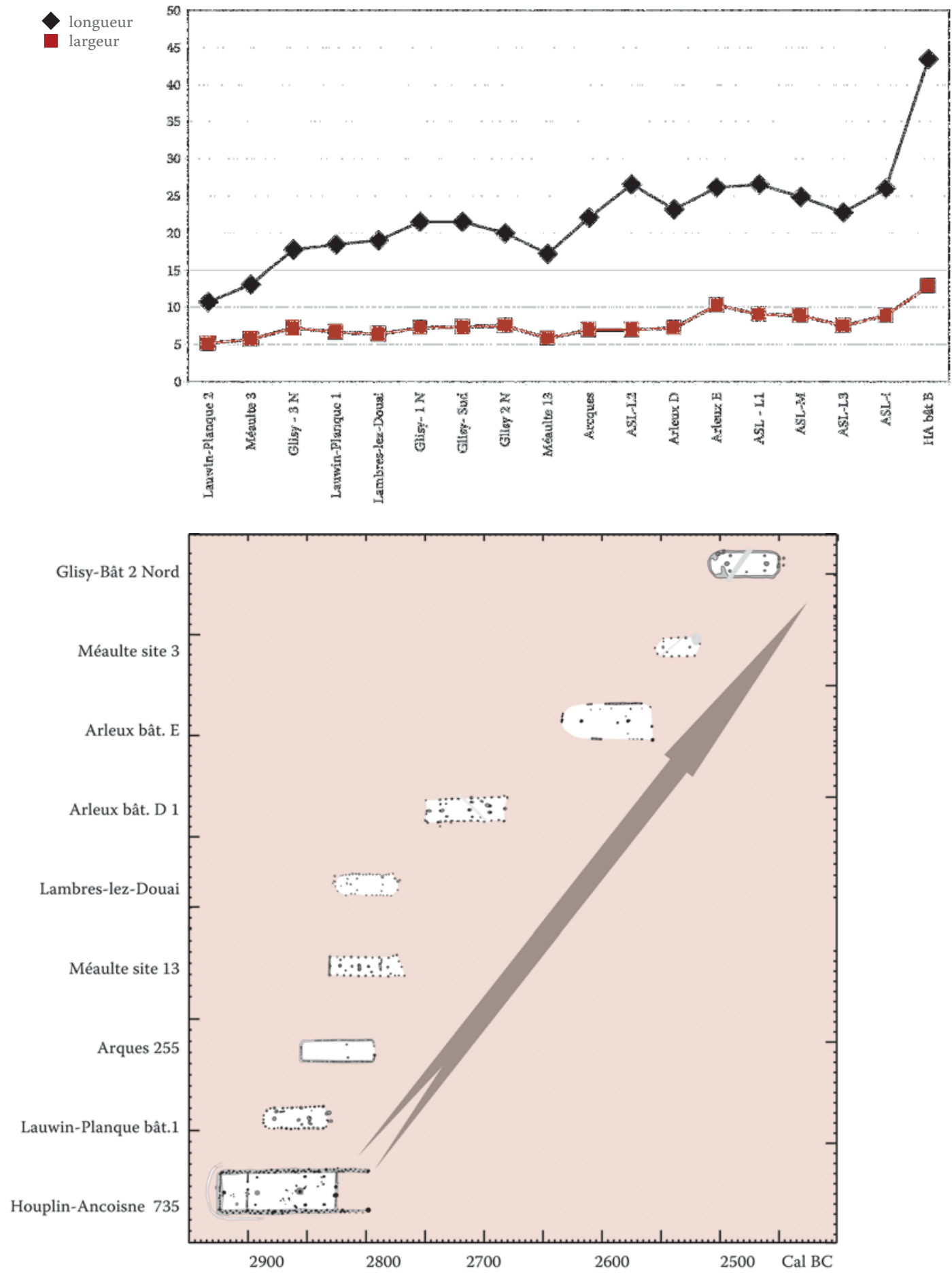

[Fig.3] Longueur et largeur des bâtiments du Néolithique final.

[Fig.5] Évolution typo-chronologique des bâtiments du groupe Deûle-Escaut

[Fig.6] Surface interne en $\mathrm{m}^{2}$ des bâtiments du Néolithique final.

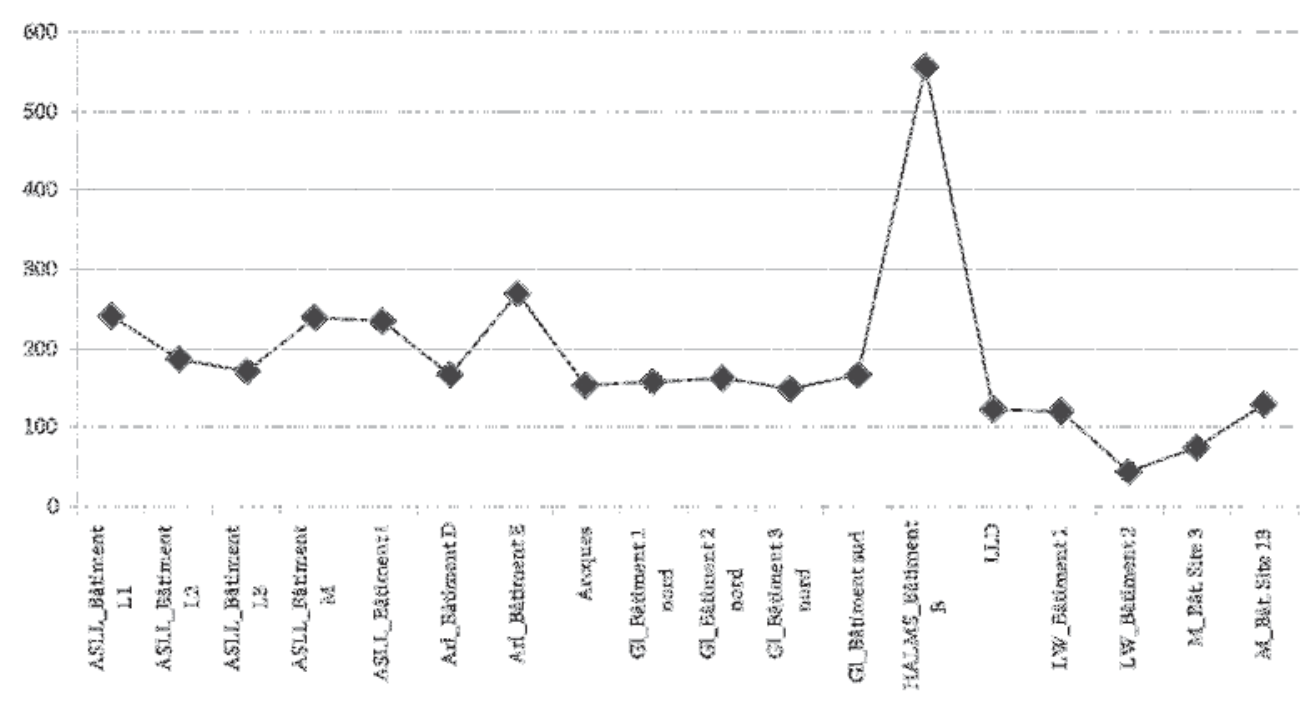


l'aménagement d'un vaste espace central et compensent les efforts de charge dû à l'écartement des poteaux faîtiers [Fig.2]. Ils sont le plus souvent implantés au sein d'une tranchée de fondation, préférée à l'avant-trou individuel ou à des fondations mixtes même si les deux solutions techniques existent. La tranchée favorise l'implantation rapprochée, voire jointive, des montants de façade. La régularité et l'alignement des montants suggèrent la présence d'une panne sablière destinée à solidariser la structure, à recevoir et à répartir les efforts exercés en rive. La quantité importante de matériaux nécessaire à la réalisation des parois externes explique vraisemblablement les diverses formes observées pour les sections des éléments d'ossature. Celles-ci résultent parfois de la refente d'un même fût de chêne en général, débité en moitié comme sur le site d'Aire-sur-la-Lys [Fig.4].

Cette communauté de caractères techniques et morphologiques signe une unité culturelle. On remarque toutefois une évolution dans la morphologie des constructions qui, confrontée aux datations absolues, a permis sur un site de proposer une évolution typo-chronologique sur un siècle environ (Glisy dans la Somme ; Joseph et al., 2011). Les maisons rectangulaires seraient, selon cette hypothèse, plus anciennes que les constructions à terminaison en abside.

Élargie à l'ensemble des données régionales, sur une durée de quatre siècles [Fig.5], la réalité semble plus complexe. Le Néolithique final régional illustre le passage d'une tradition ancienne de longs bâtiments rectangulaires à deux nefs séparées par un axe faîtier majeur vers une forme d'habitat à terminaison en abside annonçant les grandes maisons longues et étroites de l'âge du Bronze dans le Nord de l'Europe. Mais cette tendance n'explique pas la coexistence des différentes formes de l'habitat, et ce, dès le début de la période, qui pourrait témoigner alors du statut de chaque site.

Au sein du Deûle-Escaut, deux types d'organisation spatiale sont identifiés : les habitats ceinturés par une palissade de poteaux et ceux, les plus nombreux, qui se développent en aire ouverte. L'un comme l'autre livrent des bâtiments plus ou moins imposants. La diversité dans ces aménagements signale des disparités dans le statut des sites qui se manifestent principalement par l'investissement social consenti pour l'édification des structures, palissade et bâtiment, dépassant les capacités d'une unité domestique ou d'une maisonnée. Et, à y regarder de plus près, on peut se demander à quoi pouvaient servir les surfaces internes de ces habitations, souvent comprises entre 100 et $300 \mathrm{~m}^{2}$ [Fig.6], offrant ainsi de grands espaces dont la vocation reste à éclaircir. S'agissait-il de maisons collectives pouvant abriter plusieurs unités familiales ou bien de bâtiments occupant une place centrale dans le village autour duquel s'organiserait la vie communautaire ? Il est peut-être prématuré de définir précisément les relations entretenues entre les sites, mais cette variabilité architecturale pourrait rendre compte d'un réseau centralisé et hiérarchisé (Brun, 2006).
À des villages ou hameaux en milieu ouvert, comportant un grand bâtiment (entre 100 et $200 \mathrm{~m}^{2}$ ) associé à des constructions plus petites, se superposeraient des implantations caractérisées par des édifices plus importants (supérieurs à $200 \mathrm{~m}^{2}$ ) et cernées par une palissade et enfin, au-dessus de ce niveau, un site dont l'architecture ostentatoire viserait à asseoir le pouvoir politique d'une communauté sur un territoire. Cette forme d'organisation spatiale se traduit dans la composition des mobiliers associés à ces établissements. Les études pluridisciplinaires menées sur les sites de la vallée de la Deûle montrent comment les analyses paléobotaniques, technologiques et fonctionnelles des industries peuvent aider à préciser l'interdépendance des occupations au sein d'un territoire partagé (Martial et al., 2011).

BRUN P., 2006 : « Pour une archéologie des réseaux locaux. Les outils d'analyse et les problèmes d'échelles spatiales et temporelles ", in Brun P., Marcigny C., Vanmoerkerke J. (Dir.), Actes de la table ronde de Châlons-en-Champagne, 14-15 juin 2005, Les Nouvelles de l'Archéologie, n 104-105, Paris, p. 5-12.

Desbrosse V., Peltier V., 2010 : « Premiers résultats de la fouille préventive du "Haut de Launoy" à Pont-sur-Seine », Journée d'information du 20 novembre 2010, Interneo, 8, Paris.

FouÉRÉ P., 1998 : « Deux grands bâtiments du Néolithique final artenacien à Douchapt (Dordogne) », in Rencontres méridionales de Préhistoire récente (deuxième session), Arles, 1996, Antibes, éd. APDCA, , p. 311-328.

Hamon T., Hodara J.-J. :2005 : « Architectures monumentales de la fin du Néolithique, l'exemple de Moulins-Sur-Céphons (Indre) », Actes des congrès nationaux des sociétés historiques et scientifiques $\left(127^{\mathrm{e}}\right)$, Nancy, 2002, p. 299-314.

Joseph F., Julien M., Leroy-Langelin E., Lorin Y., Praud I., 2011 : "L'architecture domestique des sites du $\mathrm{III}^{\mathrm{e}}$ millenaire av. notre ère dans le Nord de la France ", in Bostyn F., Martial E., Praud I. (Dir.), Le Néolithique du Nord de la France dans son contexte européen: habitat et économie aux 4 e et ze millénaires av. notre ère, Actes du 29 e colloque interrégional sur le Néolithique Villeneuve d'Ascq, 2-3 octobre 2009, Revue Archéologique de Picardie, n spécial, 28, p. 249-274.

Lorin Y., TrawKa H., 2006 : « Le Néolithique final sur le site de la "Z.A.C. Saint-Martin" à Aire-sur-la-Lys (Pas-de-Calais) : nouvelles données sur l'architecture domestique dans le Nord de la France », Journée d'information du 18 novembre 2006, Interneo-SPF, Paris, p. 95-102.

Louboutin C., Burnez C., Constantin C., Sidéra I., 1997: «Beaumont-La Tricherie (Vienne) et Challignac (Charente) : deux sites d'habitat de la fin du Néolithique », Antiquités Nationales, 29, p. 49-62.

Martial E., Praud I., Bostyn F., 2004: « Recherches récentes sur le Néolithique final dans le Nord de la France », in VANDER Linden M., Salanova L. (DIR.), Le troisième millénaire dans le Nord de la France et en Belgique, Actes de la journée d'études SRBAP-SPF, Lille, 8 mars 2003, Société Préhistorique Française (Mémoire, XXXV), Anthropologica et Praehistorica, 115, p. 49-71.

Martial E., Cayol N., Hamon C., Maigrot Y., Medard F., MONCHABLON C., 2011 : « Production et fonction des outillages au Néolithique Final dans la vallée de la Deûle (Nord-Pas-de-Calais, France) », in Bostyn F., Martial E., Praud I. (Dir.), Le Néolithique du Nord de la France dans son contexte européen : habitat et économie aux $4^{e}$ et $3^{e}$ millénaires av. notre ère, Actes du $29^{e}$ colloque interrégional sur le Néolithique, Villeneuve d'Ascq, 2-3 octobre 20o9, Revue Archéologique de Picardie, n spécial, 28, p. 365-390

Praud I., Bernard V., Martial E., Palau R., 2007 : «Un grand bâtiment du Néolithique Final à Houplin-Ancoisne "Le Marais de Santes" (Nord, France) », in BESSE M. (DIR.), Sociétés néolithiques. Des faits archéologiques aux fonctionnements socio-économiques, Actes du 27e colloque interrégional sur le Néolithique, Neuchâtel, 1-2 octobre 2005, Cahiers d'archéologie romande, 108, p. 445-460

Pautreau J.-P., 1994: « Le grand bâtiment d'Antran (Vienne) : une nouvelle attribution chronologique », Bulletin de la Société Préhistorique Française, t. 91, n ${ }^{\circ}$, p. 418-419.

Tinévez J.-Y., 2004, Le site de la Hersonnais à Pléchâtel (Ille-et-Vilaine): un ensemble de bâtiments collectifs du Néolithique final, Travaux de la Société Préhistorique Française, n 5, 171 p. 